\title{
The Suppression of Narrow band Jammers Algorithm and the Implementation of Beidou B3 Signal
}

\author{
C.M. Li \\ School of information science and engineering \\ Hebei University of science and technology \\ Shijiazhuang, Hebei, China
}

\author{
L. Huang \\ School of information science and engineering \\ Hebei University of science and technology \\ Shijiazhuang, Hebei, China
}

\author{
X.J. Wang \\ School of information science and engineering \\ Hebei University of science and technology \\ Shijiazhuang, Hebei, China
}

\begin{abstract}
This paper investigates the use of the transform domain adaptive filtering algorithm to suppress norrowband jammers in Beidou B3 signal, which reduces the amount of calculation and improves Convergence properties compared to the time domain least mean square algorithm. The theorem and derivation process of the timeand frequency domain adaptive filters and the convergence property is discussed. Finally, analysis the frequency spectrum properties of transform domain adaptive filtering before and after suppressing of the narrowband jammers and the convergence of weights and mean square error via matlab simulation, And compare to the time domain least mean square which show that the frequency domain adaptive filtering can effectively suppress the single-tone jammer.
\end{abstract}

Keywords-the least mean square; tap weight convergence; adaptive filtering; single-tone jammer; matlab simulation

\section{INTRODUCTION}

Due to the satellite signal is weak and susceptible to interference, it is necessary to employ some type of jammer suppression technique. We proposes the transform domain adaptive filtering to suppress narrow-band jammers because of the large amount of calculation of the time domain least mean square(LMS) algorithm, which mainly studying the single-tone jammers of the narrow-band jammers. According to the characteristics of the satellite signals and jammer signals, and the energy of the jammer signals concentrated in one or some frequency bits through the Fast Fourier transform from the time domain to the frequency domain. But the useful signal has a low power spectral density in all frequency bins. The adaptive filtering should seek amplitude peaks which are indicative of a high-power jammer and suppressing jammer signals and retain the useful signal. Finally, the convergence properties of the frequency domain adaptive filter are illustrated via matlab simulation, which based on the theoretical derivation of the algorithm.

\section{The Signal Model AND the Time Domain LMS ADAPTIVE ALGORITHM}

\section{A. The Signal Model}

The received signal ,sampled at the chip rate, can be represented as[1]:

$$
x(k)=s(k)+j(k)+n(k)
$$

Where, $s(k)$ is the B3 signal of a satellite, $j(k)$ denotes the single-tone jammers, $n(k)$ is assumed to be a sample function of a white Gaussian noise.

\section{B. Derivation of the Time Domain Lms Adaptive Filtering Algorithm}

Transform domain adaptive filtering was derived based on the time domain LMS algorithm, so it is necessary to understand the time domain adaptive filtering first. The process of the LMS algorithm is to continuously adjust these weights[2-3]:

$$
\begin{gathered}
\text { Input signals: } \begin{array}{llll} 
& x(k)=\left[\begin{array}{llll}
x_{1}(k) & x_{2}(k) & \ldots & x_{n}(k)
\end{array}\right]^{T} \text { and Weight } \\
\text { signals: } & W(k)=\left[\begin{array}{llll}
w_{1}(k) & w_{2}(k) & \ldots & w_{n}(k)
\end{array}\right]^{T} \\
\text { Output } & \\
y(k)= & \sum_{i=1}^{n} w_{i}(k) x_{i}(k)=x^{T}(k) W(k)=W^{T}(k) x(k)
\end{array}
\end{gathered}
$$

The error signal: ${ }^{\varepsilon(k)=d(k)-y(k)}$, where, $d(k)$ represents the desired response

Performance function of mean square error:

$$
\xi(k)=E\left[\varepsilon^{2}(k)\right]
$$


LMS adaptive filteringalso do not require explicit measurements of correlation functions or matrix inversion, which is based on the method of steepest descent. Using the $\nabla$ represents the surface of the mean square error performance:

$$
\nabla=\frac{\partial \xi}{\partial W}=\left[\frac{\partial \xi}{\partial w_{1}(k)} \frac{\partial \xi}{\partial w_{2}(k)} \cdots \frac{\partial \xi}{\partial w_{n}(k)}\right]^{T}=2 E\left[\varepsilon(k)\left(\frac{\partial \varepsilon}{\partial w_{1}(k)} \frac{\partial \varepsilon}{\partial w_{2}(k)} \cdots \frac{\partial \varepsilon}{\partial w_{n}(k)}\right)\right]^{T}
$$

In order to obtain the weight iteration formula, it is necessary to further derivation, $-x(k)$ are the partial derivatives of the mean square error with respect to the weight values:

$$
\left[\frac{\partial \varepsilon}{\partial w_{1}(k)} \frac{\partial \varepsilon}{\partial w_{2}(k)} \cdots \frac{\partial \varepsilon}{\partial w_{n}(k)}\right]^{\mathrm{T}}=-x(k)
$$

Substituting the above equation into (2):

$$
\nabla=-2 E[\varepsilon(k) x(k)]
$$

We assume that the input signals and expected response can be regarded as stationary stochastic variables, the input signals and desired response are mutually uncorrelated, the mean-square error can be calculated as follows:

$$
\xi(k)=E\left[\varepsilon^{2}(k)\right]=E\left[d^{2}(k)\right]+W^{T}(k) E\left[x(k) x^{T}(k)\right] W(k)-2 E[d(k) x(k)] W^{T}(k)
$$

Where, the mean-square error is a quadratic function of the weight values.

We assume the $R=E\left[x(k) x^{T}(k)\right]$ is the autocorrelations of the input signals, and the column matrix $r=E[d(k) x(k)]$ is the set of cross correlations between the input signals and the desired response signal. The mean square error can also be defined as

$$
\xi(k)=E\left[\varepsilon^{2}(k)\right]=E\left[d^{2}(k)\right]+W^{T}(k) R W(k)-2 r W^{T}(k)
$$

By using the LMS algorithm for finding approximate solutions .The weight vector on the $(k+1)$ th iteration is:

$$
W(k+1)=W(k)+\mu(-\nabla)
$$

Where, ${ }^{\mu}$ is the convergence factor. According to the above equations,(7) can be expressed as:

$$
W(k+1)=W(k)+2 \mu x(k)\left[d(k)-W^{T}(k) x(k)\right]
$$

and then taking the expected value of both sides of (9),becomes:

$$
\begin{aligned}
& E[W(k+1)]=(1-2 \mu R) E[W(k)]+2 \mu r \\
& \text { The convergence of } E[W(k)] \text { can be defined } \\
& \text { as : }
\end{aligned}
$$

Where $\lambda_{\max }$ is the maximum eigenvalue of the autocorrelations of the input signals.

\section{The Derivation OF The Transform Domain ADAPTIVE FILTERINGALGORITHM}

\section{A. Frequency Domain Adaptive Filtering theory}

Frequency domain adaptive filtering process: the input signal is processed by $\mathrm{N}$ points Fourier transform for conversion into the frequency domain. A signal-tap complex adaptive filter acts on each frequency bin of the transform domain signal, then obtaining the error signal by difference between the expected signal and the weights of the output signal, and then will be processed by an inverse Fourier transform to return to the time domain, which can suppress the jammers. We assume that the error signal as the output signal. Figure 1 is the block diagram of a frequency-domain adaptive filter.

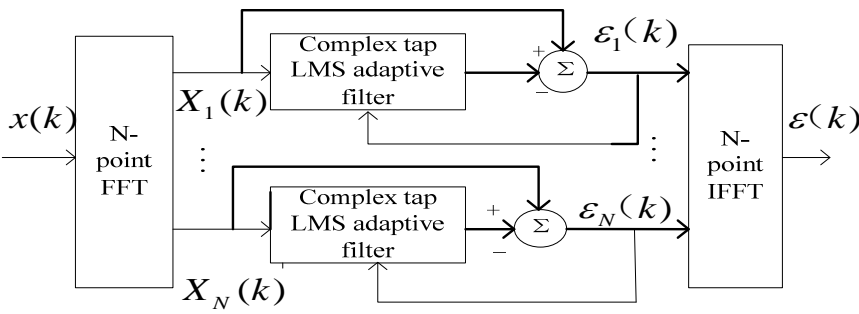

\section{FIGURE I. THE FREQUENCY ADAPTIVE FILTERS}

In the Suppression of norrowband jammers algorithm that based on FFT, in order to reduce the spectrum leakage, add the window function in every data segmentation before FFT transform in the frequency domain direct sequence spread spectrum anti-jammer algorithm.

The computation comparison: the transform-domain filter provides significant implementation advantages compared to time-domain filter[4] . Accordingly, the ratio of the frequency domain LMS for computation of time domain LMS as follows:

$$
\frac{\text { number of frequency domain multiply }}{\text { number of time domain multiply }}=\frac{3 \log _{2}(N / 2)+4}{2 N}
$$

The Fourier transforms produce a set of nearly orthogonal component, which can form autocorrelation matrix that approximate to diagonal matrix, which improved the convergence properties compared to the time domain LMS algorithm. 
B. The derivation of frequency domain adaptive filtering algorithm

As with time domain adaptive filtering, the frequency adaptive weights can be obtained by the Widrow-Hoff algorithm. As for one tap weight adaptive filter, the update weight can be described as follows:

$$
W(k+1)=W(k)+2 \mu \varepsilon(k) X^{*}(k)
$$

Where, $X(k)$ are the frequency signal, and the “*”represents the complex conjugate.

The following discussion of frequency adaptive algorithm for the suppression of narrowband jammer in spread spectrum domain[5-6]. The block diagram of frequency domain adaptive filter can be modified as following:

The input signal becomes one from two, and using $X(k)$ instead of the desired signal;

Introducing weight leakage factor $\alpha,(0<\alpha<1)$ and the weight update equation becomes:

$$
W(k+1)=\alpha W(k)+2 \mu \varepsilon(k) X^{*}(k)
$$

Making the error signal $\varepsilon(k)$ as the output signal

$$
\varepsilon(k)=X(k)-W^{T}(k) X(k)=\left[1-W^{T}(k)\right] X(k)
$$

This derivation is based on the basic Widrow-Hoff LMS algorithm. Substituting the error signal into (12), becomes:

$$
W(k+1)=\alpha W(k)+2 \mu[1-W(k)] X(k) X^{*}(k)
$$

$$
\begin{aligned}
& \text { Assuming that } X(k) \text { is independent of } \\
& X^{*}(k) \text { and } W(k) \text { is independent of } \\
& X(k) \text { and } X^{*}(k) \text {.Additionally, if the input samples are }
\end{aligned}
$$
assumed to be zero mean, $E\left\{X(k) X^{*}(k)\right\}$ can be replaced by the input signal power $\sigma^{2}$.The expected value of (15)is given by:

$$
E\{W(k+1)\}=\left(\alpha-2 \mu \sigma^{2}\right) E\{W(k)\}+2 \mu \sigma^{2}
$$

Assume that the weight equal $W(0)$ at time zero,The expected value of the $(i+1)$ th weight can be described as following (17) based on the equation above:

$$
E\{W(i+1)\}=\left(\alpha-2 \mu \sigma^{2}\right)^{i+1} W(0)+2 \mu \sum_{n=0}^{i}\left[\alpha-2 \mu \sigma^{2}\right]^{n} \sigma^{2}
$$

Consider the first term on the right, as $i \rightarrow \infty, E\{W(i)\}$ tends to a certain value, and $E\{W(i)\}$ is independent of $E\{W(0)\}$,thus, the convergence factor value should be satisfied the bound :

$$
\left|\alpha-2 \mu \sigma^{2}\right|<1
$$

Due to the convergence factor $\mu>0$,therefore, a bound is placed on ${ }^{\mu}$ to ensure that weight value is convergence:

$$
0<\mu<(\alpha+1) / 2 \sigma^{2}
$$

Consider the second term of (17) on the right equal $2 \mu \sigma^{2} /\left[1-\alpha+2 \mu \sigma^{2}\right]$ as $i \rightarrow \infty$, because it is the Geometric series summation of common ratio is $\alpha-2 \mu \sigma^{2}$ and The first item is one.In conclusion, the first term approaches zero as $i \rightarrow \infty$,then obtaining the optimized weight:

$$
W(\infty)=\sigma^{2} /\left\{[(1-\alpha) / 2 \mu]+\sigma^{2}\right\}
$$

\section{Algorithm Simulation And Performance ANALYSIS}

Frequency domain LMS algorithm is compared with the time-domain LMS computational complexity and convergence properties simulation diagram can be obtained by the formula (11) as shown in Figure 2:

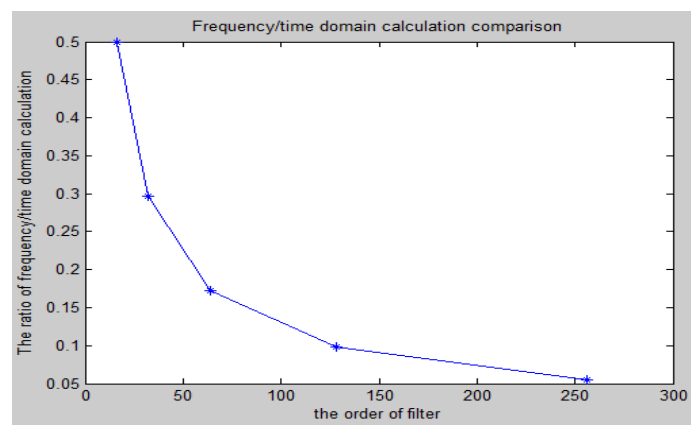

FIGURE II. FREQUENCY DOMAIN LMS COMPARED WITH TIME DOMAIN LMS COMPUTATIONAL COMPLEXITY(LEFT)

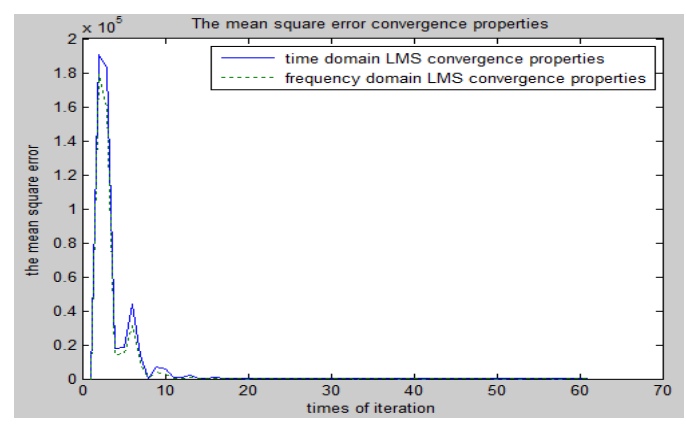

FIGURE III. FREQUENCY DOMAIN LMS COMPARED WITH TIME DOMAIN LMS CONVERGENCE PROPERTIES(RIGHT) 
Figure 2 shows that the frequency domain LMS algorithm computational complexity is greatly reduced compared with the time domain LMS algorithm.

In Figure 3, the simulation results show that the time domain LMS and frequency domain LMS have similar convergence speed under the same convergence factor when the algorithm is convergent.

The analysis of the frequency domain adaptive anti-jamming propertiesas follows: assuming signal sampling frequency:

$$
f_{s}=62 \mathrm{MHz}
$$

carrier

$$
\text { frequency: } \quad f_{0}=1268.52 \mathrm{MHz}
$$

power: $j s r=70 d B$,carrier-to-noise ratio: $C N R=37 d B$,and the tap weight leakage: $\alpha=1-2^{-14}$,By the weights of convergence condition formula(19) can calculated the convergence value: $0<\mu<1.26 \times 10^{-12}$. Here, we use 256 FFT points instead of 128 FFT points, As the general data points more, select the data section after FFT transform and the simulation analysis, adopting 61 iterations.

Add a single tone jammer at time zero, and Figure 4 shows that signal spectrum before and after suppressing the single-tone jammers under the same convergence factor. Figure 4(a), (b) respectively before and after the single tone jammer suppressed signal spectrum.

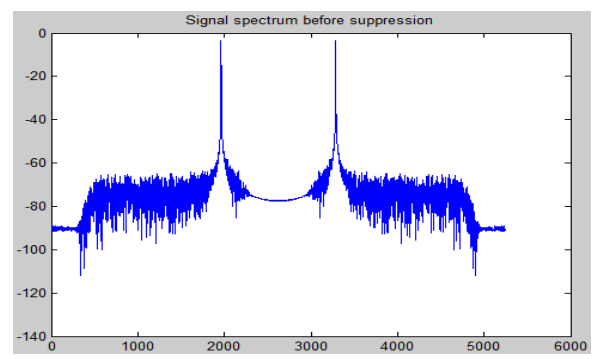

(a)Signal spectrum before suppression

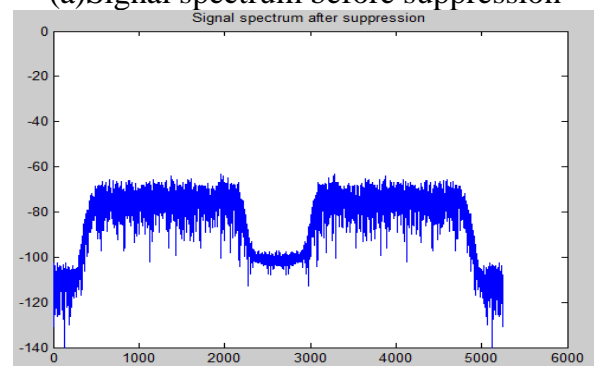

(b)Signal spectrum after suppression

FIGURE IV. SIGNAL SPECTRUM BEFORE AND AFTER SUPPRESSING SINGLE-TONE JAMMERS.

Figure 5 shows the curve of the amplitude of weights and mean square error convergence properties. Figure 5(a)(b) respectively show the amplitude of jammer frequency signal and non-jammer frequency signal for the times of iteration, Figure 5(c)shows the mean square error as a function of the times of iteration of different convergence factor. For the convergence factor ata $1=2^{-43}$,the weights of amplitude and mean square error tends to be stable after 40 iterations, and the convergence time is about $t_{2} \approx 83 u$, while the convergence factor ata $=2^{-41}$, the weights of amplitude and mean square error tends to be stable after 12 iterations, and the convergence time is about $t_{2} \approx 25 u s$. Therefore, the bigger convergence factor results in less number of iterations within a certain range of convergence factor as show in Figure 5(a)(b)(c), and the convergence rate is consistent between the jammer frequency signal and non-jammer frequency signal.

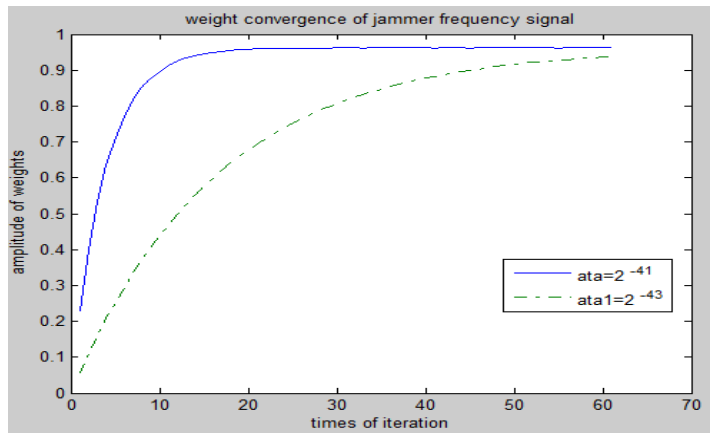

(a) Weight convergence of jammer frequency signal(left)

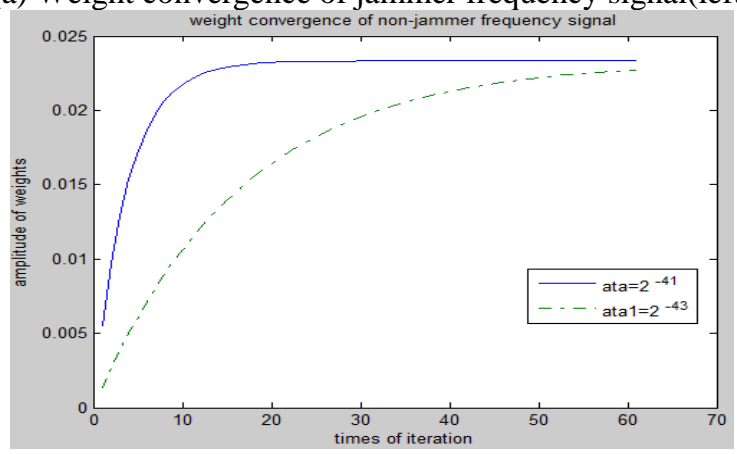

(b) Weight convergence of non-jammer frequency signal(right)

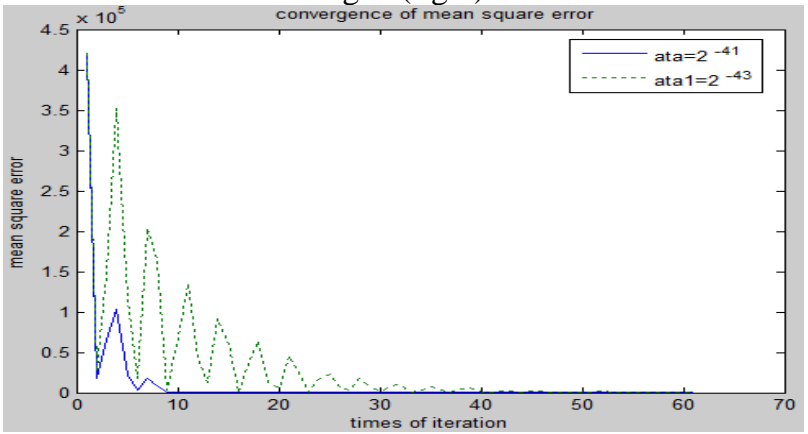

(c) Convergence of mean square error

FIGURE V. THE CONVERGENCE OF WEIGHT AND MEAN SQUARE ERROR

\section{CONCLUSIONS}

This paper mainly discusses the use of the transform domain adaptive filter for suppression of signal-tone jammer which in the beidou B3 signal. Compared with time domain LMS algorithm,the simulation results show that similar convergence rate under the same convergence factor,but the amount of calculationis reduced greatly. Simulation analysis presented in this paper show the transform domain adaptive filtering can effectively suppress the signal-tone jammer. The 
convergence time is consistent whether the jammer frequency signal or non-jammer frequency signal, and the different convergence factor results in different time.

\section{ACKNOWLEDGEMENT}

The paper is supported by 2014 Academy of Scientific Research Foundation.

\section{REFERENCES}

[1] J. W. Hsu and A. A. Giordano. Adaptive Algorithms for Estimating and Suppressing Narrow-Band Interference in PN Spread-Spectrum Systems[J]. IEEE Trans. Commun, vol. COM-30, no. 5, pp. 913-924, May 1982.

[2] Yao-huan Gong. Adaptive filter(Second edition)-Time Domain Adaptive Filtering and Smart Antennas[M]. Beijing: Publishing House of Electronics Industry, 2003.

[3] F. A. Reed and P. L. Feintuch. Acomparison of LMS Adaptive Cancelers Implemented in the Frequency Domain and the Time Domain[J]. IEEE Trans. Signal Proces, vol. ASSP-29, no. 3, pp. 770-775. June 1981

[4] Yan-xin Gou. Wireless Anti-Intercept and Anti-Jamming Communication[M]. Xian: Xidian University Press, 2011.

[5] Saulnier, G. Suppression of Norrowband Jammers in a Spread-spectrum Receiver Using Transform-Domain Adaptive Filtering[J]. IEEE Journal on Selected Areas in Communication. Vol. 10. No. 4 .may 1992

[6] Chao Tian. Study of frequency-domain block LMS Adaptive filtering Algorithm[J]. Xian: Xian university of science and technology press, 2010: $15-23$. 